University of Wollongong

Research Online

Faculty of Engineering and Information

Faculty of Engineering and Information

Sciences - Papers: Part B

Sciences

2017

Joint Low-Rank and Sparse based Image Reconstruction for Through-theWall Radar Imaging

Fok Hing Chi Tivive

University of Wollongong, tivive@uow.edu.au

Abdesselam Bouzerdoum

University of Wollongong, bouzer@uow.edu.au

Follow this and additional works at: https://ro.uow.edu.au/eispapers1

Part of the Engineering Commons, and the Science and Technology Studies Commons

Research Online is the open access institutional repository for the University of Wollongong. For further information contact the UOW Library: research-pubs@uow.edu.au 


\title{
Joint Low-Rank and Sparse based Image Reconstruction for Through-the-Wall Radar Imaging
}

\author{
Abstract \\ Through-the-wall radar uses electromagnetic waves to detect and discern targets behind opaque \\ obstacles, such as doors and walls. Wall clutter mitigation and scene reconstruction are performed to \\ produce the image of the behind-the-wall scene. These two problems, however, are often addressed \\ separately, which may result in a suboptimal solution. In this paper, the wall clutter removal and image \\ formation are unified as a joint low-rank and sparsity constrained optimization problem, which is solved \\ using augmented Lagrange multiplier method. Experimental results shows that the proposed method \\ produces clearer images than the existing method that uses a wall clutter mitigation method in \\ conjunction with backprojection method for imaging.

\section{Disciplines} \\ Engineering | Science and Technology Studies

\section{Publication Details} \\ F. Tivive \& A. Bouzerdoum, "Joint Low-Rank and Sparse based Image Reconstruction for Through-the-Wall \\ Radar Imaging," in 7th IEEE International Workshop on Computational Advances in Multi-Sensor Adaptive \\ Processing (CAMSAP), 2017, pp. 1-5.
}




\title{
Joint Low-Rank and Sparse based Image Reconstruction for Through-the-Wall Radar Imaging
}

\author{
Fok Hing Chi Tivive ${ }^{1}$ and Abdesselam Bouzerdoum ${ }^{1,2}$ \\ ${ }^{1}$ School of Electrical, Computer and Telecommunications Engineering, \\ University of Wollongong, Wollongong, Australia \\ ${ }^{2}$ College of Science and Engineering, \\ Hamad Bin Khalifa University, Doha, Qatar \\ Email: tivive@uow.edu.au, a.bouzerdoum@uow.edu.au
}

\begin{abstract}
Through-the-wall radar uses electromagnetic waves to detect and discern targets behind opaque obstacles, such as doors and walls. Wall clutter mitigation and scene reconstruction are performed to produce the image of the behind-the-wall scene. These two problems, however, are often addressed separately, which may result in a suboptimal solution. In this paper, the wall clutter removal and image formation are unified as a joint low-rank and sparsity constrained optimization problem, which is solved using augmented Lagrange multiplier method. Experimental results shows that the proposed method produces clearer images than the existing method that uses a wall clutter mitigation method in conjunction with backprojection method for imaging.
\end{abstract}

\section{INTRODUCTION}

Through-the-wall radar imaging (TWRI) is an emerging sensing technology capable of imaging behind walls and inside enclosed building structures to detect, locate, and identify moving or stationary targets. Over the past two decades, the research effort was focussed on developing inversion techniques to reconstruct the scene behind the wall. A number of imaging approaches were proposed for TWRI, such as delayand-sum (DS) beamforming (or backprojection) [1], time reversal technique [2], tomographic method [3], linear sampling method [4], and compressed sensing (CS) based methods [5]. Among them, DS beamforming is one of the methods that is commonly used for imaging, due to its simple implementation. However, most of these imaging techniques assume that the processed radar signals are free from wall clutter, which is removed using a wall clutter mitigation method, e.g., background subtraction. Furthermore, solving the wall clutter removal and image formation problems separately may result in a sub-optimal solution, where the formed image contains strong residual clutter. Therefore, techniques that jointly solve these two problems are needed to produce high quality images for target detection and classification.

There are a few approaches that have been proposed recently to solve these two problems simultaneously [6][9]. All of these methods are based on CS, where only a reduced set of measurements is available for imaging. In [6], a multi-stage approach was proposed for signal reconstruction from missing measurements, wall clutter mitigation, and image formation. Firstly, a sparse representation of the received signal is estimated from the compressed data. Then, the subspace projection method developed in [10] is employed to remove the sparse coefficients carrying the wall clutter. Finally, an $\ell_{1}$-norm optimization technique is applied to reconstruct the image from the sparse target coefficients. Ahmad et al. used prior knowledge of the wall location, wall thickness, and Fourier basis to determine an orthogonal projection matrix, which is combined with the CS image formation model [7]. Then, a greedy optimization technique, namely orthogonal matching pursuit (OMP), is applied to reconstruct the sparse target image. In [8], modulated discrete prolate spheroidal sequences (DPSS) are used to form a dictionary and the block OMP technique is applied to estimate the direct wall reflections and the wall reverberations up to $1.5 \mathrm{~m}$ behind the wall. The reconstructed wall return is then subtracted from the compressed data before applying an $\ell_{1}$-norm optimization technique for image formation. In [9], an alternative model was proposed, which combines wall clutter mitigation and CS image formation. The wall reflections are captured in a lowrank matrix, whereas the target pixels are determined by sparse coefficients. The low-rank matrix and the sparse vector are estimated by solving a constrained optimization problem.

In this paper, a low-rank and sparse model is proposed to jointly solve the wall clutter mitigation and image formation problems, without relying on prior knowledge of the interrogated TWRI scene. The rationale for using low-rank representation technique to remove the wall contribution from the radar data is that it is robust to outliers, compared to classical SVD method [11]. Contrary to the method in [9] which recovers a sparse vector from the compressed measurements for scene reconstruction, the proposed method produces a sparse matrix in which each column is a formed image at a particular antenna location. These antenna images are then weighted and combined to generate the final target image. In [12], a weight vector is added to the through-the-wall beamforming model to control the shape and sidelobe structure of the system point spread function. Here, the weight vector is estimated together with the low-rank matrix and the sparse matrix by solving the formulated optimisation problem using the augmented Lagrange multiplier technique.

The remainder of the paper is organized as follows. Section II presents briefly the signal model of TWRI and DS beamforming. Section III describes the proposed joint low-rank and sparse model for wall clutter removal and image formation, followed by experimental results and discussion in Section IV. Finally, Section V gives the conclusion. 


\section{Through-The-Wall Radar Signal Model}

A mono-static synthetic aperture radar (SAR) is considered for TWRI. At each scanning position, the transceiver emits a wideband stepped-frequency signal comprising $M$ frequencies, $\omega_{m}(m=1, \ldots, M)$. Suppose the transceiver is placed at $N$ different locations forming a linear array aperture, and there are $P$ targets behind the wall, then the radar signal $\boldsymbol{y}_{n}$ at the $n$th scanning position can be modelled as the superposition of the wall return $\boldsymbol{y}_{n}^{\mathrm{w}}$, target signal $\boldsymbol{y}_{n}^{\mathrm{t}}$, and noise $\boldsymbol{e}_{n}$ :

$$
\boldsymbol{y}_{n}=\boldsymbol{y}_{n}^{\mathrm{w}}+\boldsymbol{y}_{n}^{\mathrm{t}}+\boldsymbol{e}_{n}, \quad n=1, \ldots, N .
$$

The signals backscattered from the wall, $\boldsymbol{y}_{n}^{\mathrm{w}}$, and target, $\boldsymbol{y}_{n}^{\mathrm{t}}$, at the $m$ th frequency and $n$th antenna location can be expressed as

$$
y_{n}^{\mathrm{w}}(m)=\sum_{r=0}^{R} \sigma_{\mathrm{w}, n} A_{r} \exp \left(-j \omega_{m} \tau_{\mathrm{w}, n}^{r}\right)
$$

and

$$
y_{n}^{\mathrm{t}}(m)=\sum_{p=1}^{P} \sigma_{p} \exp \left(-j \omega_{m} \tau_{p, n}\right),
$$

where $\sigma_{\mathrm{w}, n}$ is the complex reflectivity of the wall at the $n$th scanning position, $R$ is the number of wall reverberations, $\tau_{\mathrm{w}, n}^{0}$ is the propagation delay associated with the direct return from the wall, $\tau_{\mathrm{w}, n}^{r}, r>0$ is the propagation delay associated with the $r$ th wall reverberation, $A_{r}$ is the path loss factor associated with the $r$ th wall return, $\sigma_{p}$ is the complex reflectivity of the $p$ th target, and $\tau_{p, n}$ is the two-way propagation delay between the $n$th scanning position and the $p$ th target [8].

For image formation, the interrogated scene is partitioned into a rectangular grid comprising $Q$ pixels, where the image pixels are arranged into a vector $\boldsymbol{x} \in \mathbb{C}^{Q \times 1}$. The relationship between the image obtained from the $n$th scanning position and the target signal can be expressed as

$$
\boldsymbol{x}_{n}=D_{n} \boldsymbol{y}_{n}^{\mathrm{t}}, \quad n=1, \ldots, N,
$$

where $D_{n}(q, m)=e^{j \omega_{m} \tau_{n, q}} \in \mathbb{C}^{Q \times M}$ is a mapping matrix that links the radar data at the $n$th transceiver to the image pixels and $\tau_{n, q}$ denotes the focussing delay between the $n$th transceiver and the $q$ th pixel. In DS beamforming, the image of the behind-the-wall scene $\mathrm{x}$ is obtained by summing the $N$ individual antenna images, i.e, $\mathbf{x}=1 /(N M) \sum_{n=1}^{N} \boldsymbol{x}_{n}$. Without wall clutter mitigation, the formed image contains strong clutter, which obscures the target reflections. Thus, the wall signal needs to be removed from the received signal before image formation.

\section{JOINT LOW-RANK AND SPARSE BASED IMAGE RECONSTRUCTION}

In the proposed method, the problems of wall clutter mitigation and image formation in TWRI are addressed jointly using a joint low-rank and sparse (JLRS) model. The JLRS model captures the wall reflections in a low-rank matrix and imposes joint sparsity on images formed at different antenna locations. Let $Y$ denote the matrix containing the different antenna signals $\boldsymbol{y}_{n}$ as columns. Likewise, we denote by $Y^{\mathrm{w}}$, $Y^{\mathrm{t}}$ and $E$, the matrices comprising the wall returns, the target signals, and the noise components. Equation (1) can be written in matrix form as

$$
Y=Y^{\mathrm{w}}+Y^{\mathrm{t}}+E .
$$

Since the wall reflections are expected to be uniform across the antenna array, they are represented using a dictionary $\Phi$ and a low-rank matrix $L$,

$$
Y^{\mathrm{w}}=\Phi L .
$$

Let $X$ denote the matrix containing the target images at different locations as columns, $X=\left[\boldsymbol{x}_{1}, \ldots, \boldsymbol{x}_{N}\right]$, where $\boldsymbol{x}_{n}$ is given in (4). The final image, $\mathbf{x}$, is obtained as a weighted average of the individual antenna images, i.e., it is the fusion of the different antenna images:

$$
\mathbf{x}=X \boldsymbol{w}=\sum_{i=1}^{N} w_{i} \boldsymbol{x}_{i},
$$

where $\boldsymbol{w}=\left[w_{1}, \ldots, w_{N}\right]^{T}$ is a weight vector and ${ }^{T}$ denotes the transpose operator. To capture the wall reflections in the low-rank component and obtain a clear target image in the sparse component, low-rank and sparsity constraints are imposed on $L$ and $X$, respectively. Thus, the JLRS model can be formulated as

$$
\begin{gathered}
\min _{L, Y^{\mathrm{t}}, X, \boldsymbol{w}}\|L\|_{*}+\lambda_{1}\|X \boldsymbol{w}\|_{1}+\lambda_{2}\left\|X^{H}\right\|_{2,1} \\
+\frac{1}{2} \sum_{n=1}^{N}\left\|\boldsymbol{x}_{n}-D_{n} \boldsymbol{y}_{n}^{\mathrm{t}}\right\|_{2}^{2}+\frac{1}{2}\left\|Y-\Phi L-Y^{\mathrm{t}}\right\|_{F}^{2} \\
\text { s.t. } \mathbf{1}^{T} \boldsymbol{w}=1,
\end{gathered}
$$

where ${ }^{H}$ denotes the Hermitian transpose and $\mathbf{1}$ is a vector of all-ones. Minimizing the nuclear norm in the first term produces a low-rank representation, the second term enforces sparsity of the final composite image, and the $\ell_{2,1}$-norm in the third term imposes row sparsity on the matrix $X$.

Two auxiliary variables $J$ and $r$ are introduced to make (8) separable. Thus, the constrained optimisation problem can be rewritten as

$$
\begin{gathered}
\min _{J, L, Y^{\mathrm{t}}, X, \boldsymbol{r}, \boldsymbol{w}}\|J\|_{*}+\lambda_{1}\|\boldsymbol{r}\|_{1}+\lambda_{2}\left\|X^{H}\right\|_{2,1} \\
+\frac{1}{2} \sum_{n=1}^{N}\left\|\boldsymbol{x}_{n}-D_{n} \boldsymbol{y}_{n}^{\mathrm{t}}\right\|_{2}^{2}+\frac{1}{2}\left\|Y-\Phi L-Y^{\mathrm{t}}\right\|_{F}^{2} \\
\text { s.t. } \mathbf{1}^{T} \boldsymbol{w}=1, X \boldsymbol{w}=\boldsymbol{r}, L=J .
\end{gathered}
$$

Let $B$ and $\boldsymbol{b}$ denote the Lagrange multipliers and $\mu$ be a penalty parameter $(\mu>0)$. The inexact ALM proposed by Lin et al. [13] is employed to solve (9) by alternately minimising the following subproblems:

$$
\begin{gathered}
\min _{J}\|J\|_{*}+\frac{\mu^{k}}{2}\left\|L^{k}-J+\frac{B^{k}}{\mu^{k}}\right\|_{F}^{2}, \\
\min _{L} \frac{\mu^{k}}{2}\left\|L-J^{k+1}+\frac{B^{k}}{\mu^{k}}\right\|_{F}^{2}+\frac{1}{2}\left\|Y-\Phi L-\left(Y^{\mathrm{t}}\right)^{k}\right\|_{F}^{2}, \\
\min _{\boldsymbol{y}_{n}^{\mathrm{t}}} \frac{1}{2} \sum_{n=1}^{N}\left\|\boldsymbol{y}_{n}-\Phi \boldsymbol{l}_{n}^{k+1}-\boldsymbol{y}_{n}^{\mathrm{t}}\right\|_{2}^{2}+\frac{1}{2} \sum_{n=1}^{N}\left\|\boldsymbol{x}_{n}^{k}-D_{n} \boldsymbol{y}_{n}^{\mathrm{t}}\right\|_{2}^{2},
\end{gathered}
$$




$$
\begin{gathered}
\min _{X} \lambda_{2}\left\|X^{H}\right\|_{2,1}+\frac{\mu^{k}}{2}\left\|X \boldsymbol{w}^{k}-\boldsymbol{r}^{k}+\frac{\boldsymbol{b}^{k}}{\mu^{k}}\right\|_{2}^{2}+ \\
\frac{1}{2} \sum_{n=1}^{N}\left\|\boldsymbol{x}_{n}-D_{n}\left(\boldsymbol{y}_{n}^{\mathrm{t}}\right)^{k+1}\right\|_{2}^{2}, \\
\min _{\boldsymbol{r}} \lambda_{1}\|\boldsymbol{r}\|_{1}+\frac{\mu^{k}}{2}\left\|X^{k+1} \boldsymbol{w}^{k}-\boldsymbol{r}+\frac{\boldsymbol{b}^{k}}{\mu^{k}}\right\|_{2}^{2}, \\
\min _{\boldsymbol{w}} \frac{\mu^{k}}{2}\left\|X^{k+1} \boldsymbol{w}-\boldsymbol{r}^{k+1}+\frac{\boldsymbol{b}^{k}}{\mu^{k}}\right\|_{2}^{2}+\iota_{C}(\boldsymbol{w})
\end{gathered}
$$

where $\iota_{C}(\boldsymbol{w})$ is the indicator function of $C$ defined by $\iota_{C}(\boldsymbol{w})=0$ if $\boldsymbol{w} \in C ;+\infty$ otherwise, where $C=\{\boldsymbol{w} \in$ $\left.\mathbb{R}^{N}: \sum_{i=1}^{N} w_{i}=1\right\}$. All the above subproblems can be solved individually.

Subproblem (10) is a least squares problem regularised by a nuclear norm penalty. It can be efficiently solved using singular value shrinkage [14]. Let the shrinkage operator be defined by

$$
\mathcal{T}(\alpha, \beta)=\operatorname{sgn}(\alpha) \max (|\alpha|-\beta, 0),
$$

where $\operatorname{sgn}(\cdot)$ denotes the signum function. This shrinkage operator is applied entrywise; it can be applied to vectors as well as matrices. The minimisation of Subproblem (10) can be performed by applying the shrinkage operation as follows:

$$
\begin{aligned}
\operatorname{SVD}\left(L^{k}+B^{k} / \mu^{k}\right) & =U \Sigma V^{H}, \\
\widehat{\Sigma} & =\mathcal{T}\left(\Sigma, 1 / \mu^{k}\right), \\
J^{k+1} & =U \widehat{\Sigma} V^{H},
\end{aligned}
$$

where $U$ and $V$ are unitary matrices, and $\Sigma$ is a diagonal matrix of singular values.

Subproblem (11) is a least squares problem, which can be solved efficiently using a conjugate gradient method. Differentiating (11) with respect to $L$ and setting the gradient equal to zero yields the following solution:

$L^{k+1}=\left[\mu^{k} I+\Phi^{H} \Phi\right]^{-1}\left[\mu^{k}\left(J^{k+1}-\frac{B^{k}}{\mu^{k}}\right)+\Phi^{H}\left(Y-\left(Y^{\mathrm{t}}\right)^{k}\right)\right]$,

where $I$ denotes the identity matrix. Similarly, the solution to Subproblem (12) can be written as

$$
\left(\boldsymbol{y}_{n}^{\mathrm{t}}\right)^{k+1}=\left[I+D_{n}^{H} D_{n}\right]^{-1}\left[\boldsymbol{y}_{n}-\Phi \boldsymbol{l}_{n}^{k+1}+D_{n}^{H} \boldsymbol{x}_{n}^{k}\right] \forall n
$$

Subproblem (13) can be regarded as group LASSO (least absolute shrinkage and selection operator). Let $\quad G^{k+1}=\left[D_{1}\left(\boldsymbol{y}_{1}^{\mathrm{t}}\right)^{k+1}, \ldots, D_{N}\left(\boldsymbol{y}_{N}^{\mathrm{t}}\right)^{k+1}\right] \quad$ and $\boldsymbol{f}^{k}=\boldsymbol{r}^{k}-\boldsymbol{b}^{k} / \mu^{k}$ and $W^{k}=\boldsymbol{w}^{k}\left(\boldsymbol{w}^{k}\right)^{T}+I / \mu^{k}$. The last two quadratic functions in (13) can be simplified as

$$
\begin{aligned}
& \frac{\mu^{k}}{2}\left\|X \boldsymbol{w}^{k}-\boldsymbol{r}^{k}+\frac{\boldsymbol{b}^{k}}{\mu^{k}}\right\|_{2}^{2}+\frac{1}{2} \sum_{n=1}^{N}\left\|\boldsymbol{x}_{n}-D_{n}\left(\boldsymbol{y}_{n}^{\mathrm{t}}\right)^{k+1}\right\|_{2}^{2} \\
& =\frac{\mu^{k}}{2}\left\|X \boldsymbol{w}^{k}-\boldsymbol{f}^{k}\right\|_{2}^{2}+\frac{1}{2}\left\|X-G^{k+1}\right\|_{2}^{2} \\
& =\frac{\mu^{k}}{2} \operatorname{Tr}\left(\left(X \boldsymbol{w}^{k}-\boldsymbol{f}^{k}\right)^{H}\left(X \boldsymbol{w}^{k}-\boldsymbol{f}\right)+\frac{\left(X-G^{k+1}\right)^{H}\left(X-G^{k+1}\right)}{\mu^{k}}\right) \\
& =\frac{\mu^{k}}{2} \operatorname{Tr}\left(\left(X^{H} X-2 X^{H}\left[\boldsymbol{f}^{k}\left(\boldsymbol{w}^{k}\right)^{T}+\frac{G^{k+1}}{\mu^{k}}\right]\right)\left(W^{k}\right)^{-1}+\mathcal{C}\right) \\
& =\frac{\mu^{k}}{2}\left\|X-\left(\boldsymbol{f}^{k}\left(\boldsymbol{w}^{k}\right)^{T}+\frac{G^{k+1}}{\mu^{k}}\right)\left(W^{k}\right)^{-1}\right\|_{2}^{2}+\mathcal{C}
\end{aligned}
$$

where $\mathcal{C}$ is a constant term that is independent of the variable $X$. Let $Z^{k+1}=\left(\boldsymbol{f}^{k}\left(\boldsymbol{w}^{k}\right)^{T}+G^{k+1} / \mu^{k}\right)\left(W^{k}\right)^{-1}, Z^{k+1}(j,:)$ denote the $j$-th row of matrix $Z^{k+1}$. The objective function (13) can be rewritten as

$$
\min _{X} \lambda_{2}\left\|X^{H}\right\|_{2,1}+\frac{\mu^{k}}{2}\left\|X-Z^{k+1}\right\|_{2}^{2}
$$

and its solution can be obtained using a row-shrinkage operator [15] as

$$
X^{k+1}(j,:)=\frac{Z^{k+1}(j,:)}{\left\|Z^{k+1}(j,:)\right\|_{2}} \max \left(\left\|Z^{k+1}(j,:)\right\|_{2}-\frac{\lambda_{2}}{\mu^{k}}, 0\right) .
$$

Similarly, the solution to the $\ell_{1}$-norm regularised least squares problem (14) can be obtained by applying the shrinkage operator given in (16):

$$
\boldsymbol{r}^{k+1}=\mathcal{T}\left(X^{k+1} \boldsymbol{w}^{k}+\boldsymbol{b}^{k} / \mu^{k}, \lambda_{1} / \mu^{k}\right) .
$$

The last Subproblem (15) is a sum-to-one constrained least squares problem. Let $A^{k+1}=\left[\left(X^{k+1}\right)^{H}\left(X^{k+1}\right)\right]^{-1}, \alpha^{k+1}=$ ${ }_{1}^{T} A^{k+1} \mathbf{1}$, and $\boldsymbol{d}^{k+1}=A^{k+1}\left(X^{k+1}\right)^{H}\left[-\boldsymbol{r}^{k+1}+\boldsymbol{b}^{k} / \mu^{k}\right]$. Its solution can be written as [16]

$$
\boldsymbol{w}^{k+1}=A^{k+1} \mathbf{1}\left(\alpha^{k+1}\right)^{-1}\left(1+\mathbf{1}^{T} \boldsymbol{d}^{k+1}\right)-\boldsymbol{d}^{k+1} .
$$

The Lagrange multipliers and the penalty parameter are updated as follows [13]:

$$
\begin{aligned}
B^{k+1} & =B^{k}+\mu^{k}\left(L^{k+1}-J^{k+1}\right), \\
\boldsymbol{b}^{k+1} & =\boldsymbol{b}^{k}+\mu^{k}\left(X^{k+1} \boldsymbol{w}^{k+1}-\boldsymbol{r}^{k+1}\right), \\
\mu^{k+1} & =\min \left(\rho \mu^{k}, \mu_{\max }\right) .
\end{aligned}
$$

Here, $\rho=1.1$ and $\mu_{\max }=10^{10}$.

\section{EXPERIMENTAL RESULTS}

The proposed method is tested on real radar data collected at the radar imaging laboratory of the Centre for Signal and Information Processing (CSIP), University of Wollongong, Australia. Two baseline methods are implemented and tested. The first method applies background subtraction to remove the wall contribution and DS beamforming to form the image of the scene. The second method employs the subspace projection method [10] for wall clutter mitigation and DS beamforming for image formation. The rationale for the latter technique is that it is based on similar notion as the proposed method, i.e., capturing the wall reflections in a low-rank subspace. However, it performs wall clutter mitigation and image formation separately. The experimental setup is presented in Subsection IV-A, followed by the experimental results and discussion in Subsection IV-B.

\section{A. Experimental Setup}

A hollow wooden wall of thickness $0.08 \mathrm{~m}$ was placed at a standoff distance of $1.05 \mathrm{~m}$ in front of a mono-static throughthe-wall radar. In the scene, a metal plate and a dihedral were placed at $(-0.5,1.9) \mathrm{m}$ and $(0.8,1.0) \mathrm{m}$ behind the wall. A 31-element array aperture was synthesised to image the scene using a stepped-frequency signal of bandwidth $3 \mathrm{GHz}$ centered at $2.5 \mathrm{GHz}$ with step size of $3.75 \mathrm{MHz}$. The downrange and cross-range of the image scene were set to $3 \mathrm{~m}$, and the scene was partitioned into an image of $100 \times 100$ pixels. In 
the proposed JLRS method, the regularisation parameters $\lambda_{1}$ and $\lambda_{2}$ were computed as $\lambda_{1}=c_{1} / \sqrt{\max (M, N)}$ [17] and $\lambda_{2}=3 /\left(7 \sqrt{c_{2} \max (M, N)}\right)$ [18], where $c_{1}$ and $c_{2}$ were set to 8.5 and 0.02 , respectively. For the low-rank representation, the atom of the dictionary $\Phi$ was defined as Fourier basis. The JLRS method terminated when the relative difference between the current and previous formed images was less than a predefined threshold, i.e., $\left\|\boldsymbol{r}^{k+1}-\boldsymbol{r}^{k}\right\|_{2} /\left\|\boldsymbol{r}^{k}\right\|_{2} \leq 0.001$. To quantify the image produced by the image formation method, the improvement factor (IF) in terms of the target-to-clutter ratio (TCR) is computed as:

$$
\mathrm{IF}=10 \log \left(\frac{\mathrm{TCR}_{o}}{\mathrm{TCR}_{i}}\right),
$$

where $\mathrm{TCR}_{o}$ and $\mathrm{TCR}_{i}$ are, respectively, the target-to-clutter ratios of the formed image with and without wall clutter mitigation. The TCR of the formed image is calculated as

$$
\mathrm{TCR}=\frac{\frac{1}{N_{t}} \sum_{j \in A_{t}}\left|x_{j}\right|^{2}}{\frac{1}{N_{c}} \sum_{j \in A_{c}}\left|x_{j}\right|^{2}},
$$

where $A_{t}$ is the selected target region, $A_{c}$ is the clutter region defined as the entire image excluding the target region, $N_{c}$ and $N_{t}$ are, respectively, the number of pixels in the clutter and target regions.

\section{B. Results and Discussion}

The JLRS method and the baseline methods are tested on the same radar data. Table I lists the IFs of the three image formation methods. The JLRS method achieves higher IF than the baseline methods; it outperforms the background subtraction and subspace projection methods by $20.2 \mathrm{~dB}$ and $19 \mathrm{~dB}$, respectively. Figure 1 illustrates the images obtained by different image formation methods. Figures 1(a) and (b) show the images produced by DS-beamforming before and after background subtraction. Figures 1(c) and (d) depict the images obtained from the subspace projection method and formed by the JLRS method, respectively. It is clear that the JLRS method not only suppresses the strong wall clutter, but also reduces the background clutter in the scene.

TABLE I. IMPROVEMENT FACTOR IN TERMS OF TARGET-TO CLUTTER RATIO FOR DIFFERENT IMAGE FORMATION METHODS.

\begin{tabular}{|l|c|}
\hline Image formation method & Improvement Factor $(\mathrm{dB})$ \\
\hline JLRS method & 25.4 \\
\hline DS beamforming + Subspace method & 5.7 \\
\hline DS beamforming + Background subtraction & 4.5 \\
\hline
\end{tabular}

\section{CONCLUSiON}

In this paper, a joint low-rank and sparse model was proposed that unifies wall clutter mitigation and scene reconstruction for TWRI. The proposed model is formulated as a constrained optimisation problem to estimate a low-rank matrix capturing the wall contribution, a sparse matrix containing the individual antenna images, and a set of weights to aggregate the antenna images into the final target image. Experiments using real radar data were conducted, and the experimental results show that the proposed joint wall clutter mitigation and image formation method produces radar images with better improvement factor in terms of target-to-clutter ratio than DS beamforming in conjunction with background subtraction or subspace projection method for wall clutter mitigation.

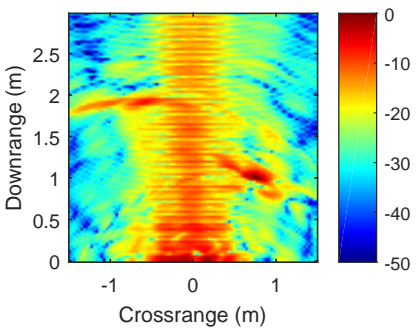

(a)

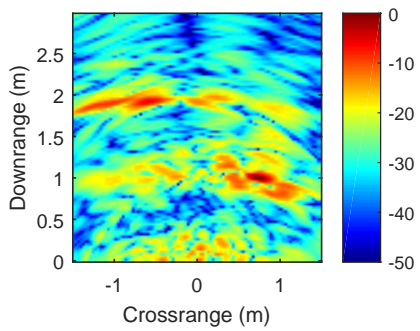

(c)

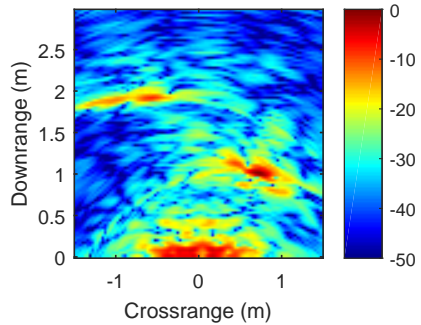

(b)

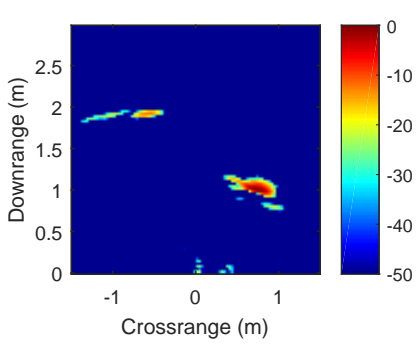

(d)
Fig. 1. TWRI images obtained using (a) DS beamforming before wall clutter mitigation, (b) DS beamforming with background subtraction, (c) DS beamforming with subspace projection, and (d) proposed JLRS method.

\section{ACKNOWLEDGMENT}

This work is supported by a grant from the Australian Research Council (ARC).

\section{REFERENCES}

[1] F. Ahmad, G. Frazer, S. Kassam, and M. Amin, "Design and implementation of near-field, wideband synthetic aperture beamformers," IEEE Transactions on Aerospace and Electronic Systems, vol. 40, no. 1, pp. 206-220, 2004.

[2] W. Zheng, Z. Zhao, and Z.-P. Nie, "Application of TRM in the UWB through wall radar," Progress in Electromagnetics Research B, vol. 87, pp. 279-296, 2008.

[3] F. Soldovieri, R. Solimene, and G. Prisco, "A multiarray tomographic approach for through-wall imaging," IEEE Transactions on Geoscience and Remote Sensing, vol. 46, no. 4, pp. 1192-1199, 2008.

[4] I. Catapano and L. Crocco, "Linear sampling method for time-lapse qualitative through-the-wall imaging," European Microwave Conference, pp. 1884-1887, Rome, Italy, 2009

[5] Y.-S. Yoon and M. G. Amin, "Compressed sensing technique for highresolution radar imaging," Proc. SPIE Vol 6968, Signal Processing, Sensor Fusion, and Target Recognition XVII, pp. 1-10, Orlando, FL, USA, 2008.

[6] F. H. C. Tivive, A. Bouzerdoum, and V. H. Tang, "Multi-stage compressed sensing and wall clutter mitigation for through-the-wall radar image formation," IEEE 8th Sensor Array and Multichannel Signal Processing Workshop (SAM), pp. 489-492, A Coruna, Spain, 2014.

[7] F. Ahmad, M. G. Amin, and T. Dogaru, "Partially sparse imaging of stationary indoor scenes," EURASIP Journal on Advances in Signal Processing, vol. 2014, no. 100, pp. 1-15, 2014. 
[8] F. Ahmad, J. Qian, and M. G. Amin, "Wall clutter mitigation using discrete prolate spheroidal sequences for sparse reconstruction of indoor stationary scenes," IEEE Transactions on Geoscience and Remote Sensing, vol. 53, no. 3, pp. 1549-1557, 2015.

[9] V. H. Tang, A. Bouzerdoum, S. L. Phung, and F. H. C. Tivive, "Radar imaging of stationary indoor targets using joint low-rank and sparsity constraints," IEEE International Conference on Acoustics, Speech and Signal Processing, pp. 1412-1216, Shanghai, China, 2016.

[10] F. H. C. Tivive, A. Bouzerdoum, and M. G. Amin, "A subspace projection approach for wall clutter mitigation in through-the-wall radar imaging," IEEE Transactions on Geoscience and Remote Sensing, vol. 53, no. 4, pp. 2108-2122, 2015.

[11] G. Liu, Z. Lin, S. Yan, J. Sun, Y. Yu, and Y. Ma, "Robust recovery of subspace structures by low-rank representation," IEEE Transactions on Pattern Analysis and Machine Intelligence, vol. 33, no. 1, pp. 171-184, 2013.

[12] M. G. Amin and F. Ahmad, "Wideband synthetic aperture beamforming for through-the-wall imaging," IEEE Signal Processing Magazine, vol. 25, no. 4, pp. 110-113, 2008.

[13] Z. Lin, M. Chen, L. Wu, and Y. Ma, "The augmented Lagrange multiplier method for exact recovery of corrupted low-rank matrices," University of Illinois, Tech. Report No. UILU-ENG-09-2215, 2009.

[14] J.-F. Cai, E. J. Candes, and Z. Shen, "A singular value thresholding algorithm for matrix completion," SIAM Journal on Optimization, vol. 20, no. 4, pp. 1956-1982, 2010.

[15] W. Deng, W. Yin, and Y. Zhang, "Group sparse optimization by alternating direction method," Proc. of SPIE Vol. 8858, Wavelets and Sparsity XV, pp. 88 580R-1 - 88 580R-15, San Diego, California, USA, 2013.

[16] D. C. Heinz and C.-I. Chang, "Fully constrained least squares linear spectral mixture analysis method for material quantification in hyperspectral imagery," IEEE Transactions on Geoscience and Remote Sensing, vol. 39, no. 3, pp. 529-545, 2001.

[17] M. P. Masarik, J. Burns, B. T. Thelen, J. Kelly, and T. C. Havens, "GPR anomaly detection with robust principal component analysis," in Proc SPIE, Detection and Sensing of Mines, Explosive Objects, and Obscured Targets XX, vol. 9454, 2015, pp. 945414 1- 94541411.

[18] H. Xu, C. Caramanis, and S. Sanghavi, "Robust PCA via outlier pursuit," IEEE Transactions on Information Theory, vol. 58, no. 5, pp 3047-3064, 2012. 